\title{
Magnetic Array Assisted Triboelectric Nanogenerator Sensor for Real-Time Gesture \\ Interaction
}

Cite as

Nano-Micro Lett.

(2021) 13:51

Received: 23 September 2020

Accepted: 30 November 2020

Published online: 5 January 2021

(C) The Author(s) 2021

\author{
Ken Qin ${ }^{1}$, Chen Chen $^{1}$, Xianjie $\mathrm{Pu}^{1}{ }^{凶}$, Qian Tang ${ }^{1}{ }^{凶}$, Wencong He ${ }^{1}$, Yike Liu ${ }^{1}$, \\ Qixuan Zeng ${ }^{1}$, Guanlin $\mathrm{Liu}^{2}$, Hengyu Guo ${ }^{1}$, Chenguo $\mathrm{Hu}^{1} \bowtie$
}

\section{HIGHLIGHTS}

- By counting the positive/negative pulses in unit time to sense the degree, speed, and direction of finger motion in real-time.

- The magnetic array assisted sliding structure translates sliding motion into contact-separation thus improves the durability and lowspeed signal amplitude.

- The magnetic track constrains the sliding direction that greatly improves the stability.

\begin{abstract}
In human-machine interaction, robotic hands are useful in many scenarios. To operate robotic hands via gestures instead of handles will greatly improve the convenience and intuition of human-machine interaction. Here, we present a magnetic array assisted sliding triboelectric sensor for achieving a real-time gesture interaction between a human hand and robotic hand. With a finger's traction movement of flexion or extension, the sensor can induce positive/negative pulse signals. Through counting the pulses in unit time, the degree, speed, and direction of finger motion can be judged in realtime. The magnetic array plays an important role in
\end{abstract}
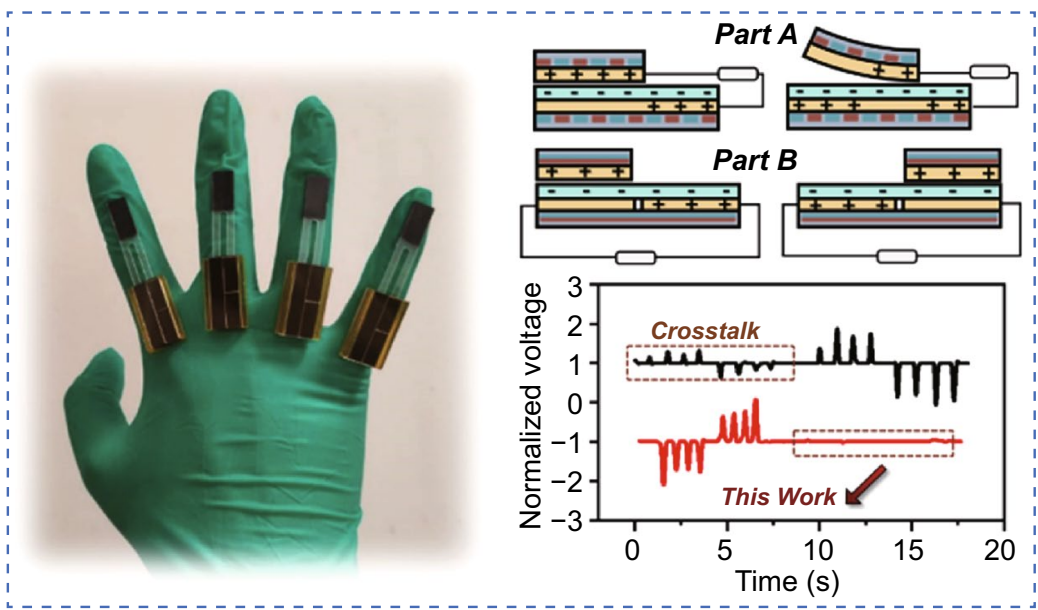
generating the quantifiable pulses. The designed two parts of magnetic array can transform sliding motion into contact-separation and constrain the sliding pathway, respectively, thus improve the durability, low speed signal amplitude, and stability of the system. This direct quantization approach and optimization of wearable gesture sensor provide a new strategy for achieving a natural, intuitive, and real-time human-robotic interaction.

KEYWORDS Sliding triboelectric sensor; Magnetic array; Gesture; Real-time; Human-machine interaction

Ken Qin and Chen Chen have contributed equally to this work

$\triangle$ Xianjie Pu, xjpu@cqu.edu.cn; Qian Tang, tangqian@cqu.edu.cn; Chenguo Hu, hucg@cqu.edu.cn

1 Department of Applied Physics, State Key Laboratory of Power Transmission Equipment \& System Security and New Technology, Chongqing Key Laboratory of Soft Condensed Matter Physics and Smart Materials, Chongqing University, Chongqing 400044, People's Republic of China

2 Center On Nanoenergy Research, School of Physical Science and Technology, Guangxi University, Nanning, Guangxi 530004,

People's Republic of China 


\section{Introduction}

Intuitive human-machine interaction (HMI) plays more and more an important role in duties and daily life. From traditional keyboards to various touchpad nowadays, HMIs have been developed to be more natural [1], integrated [2], portable [3, 4], and even wearable [5, 6]. For the skillful robotic hand in industry, military service, surgery, or entertainment, traditional interaction method based on handles cannot meet current requirements [7, 8]. A wearable gesture sensor that can perceive the dexterous movement of each finger will fulfil this need [9-12]. The emergent triboelectric nanogenerator (TENG) exhibits great potential as an alternative scheme in some fields, including physiological signal collection [13, 14], mechanical signal detecting [15, 16], and visual tracking $[8,17]$. Based on the coupling of triboelectrification and electrostatic induction, TENG has been applied in energy harvesting $[18,25,26]$ and self-powered mechanical sensing [1, 19-24]. However, most studies in gesture sensing based on TENG take signal amplitude as feature to represent finger's once time movement and ignore the intermediate process $[13,14,16]$. Moreover, to quantify the bending degree via signal amplitude is unstable, because there are too many factors affecting the amplitude. Therefore, a finger-wearable TENG sensor for quantifying the finger's bending/straightening for a real-time gesture interaction is expected [15, 27].

In this work, we present a magnetic array assisted sliding triboelectric sensor (Ma-s-TS) to achieve a real-time gesture interaction between a human hand and robotic hand. During the bending of the finger, as shown in Fig. S1, the rotation angle is proportional to the tensile displacement at the joint (as the fulcrum). Therefore, We can judge the bending degree by the sliding displacement. The basic sensing principle of Ma-s-TS is to induce positive/negative pulses under the finger traction movement (flexion/extension), and then by counting the pulses in unit time, to sense the degree, speed and direction of a finger motion in real-time. It is a creative design that the magnetic array assisted sliding structure can constrain the sliding pathway and translate the sliding motion into contact-separation, and thus can improve the stability, durability and low speed signal amplitude. This work brings an optimized scheme for real-time gesture interaction based on a wearable TENG sensor, and it promises a widespread application of intuitive, natural HMIs.

\section{Methods}

\subsection{Fabrication of Ma-s-TS}

Typically, the Ma-s-TS was fabricated as two main parts, stator and slider. The stator $\left(20 \times 12 \mathrm{~mm}^{2}\right)$ was made up of an electrode layer (thickness: $0.01 \mathrm{~mm}$ ) sandwiched between upper layer of polytetrafluoroethylene (PTFE) film (thickness: $0.08 \mathrm{~mm}$ ) and bottom layer of flexible halbach magnetic arrays made of strontium ferrite magnetic powder and rubber (thickness: $0.315 \mathrm{~mm}$ ), which was pasted on an acrylic board. The slider (length: $10 \mathrm{~mm}$, width: $20 \mathrm{~mm}$ ) was a Y-shaped PET strip, which covered a layer of flexible rubber and a layer of halbach magnetic arrays. Finally, a copper layer $(0.02 \mathrm{~mm})$ was pasted on the surface of the as-fabricated flexible rubber as the positive tribomaterial by polyimide tape. It should be noted that both the magnetic rubbers on the stator and slider were divided into two parts (part I and part II). The magnetic array of part I is perpendicular to the sliding direction, while that of Part II is parallel to the sliding direction, by which the two tribo-layers were alternately pushed up and down in sliding process.

\subsection{Characterization and Measurement}

For electric signal test of the Ma-s-TS, a home-made hinge component [15] was fabricated to simulate the finger's motion. A numerical controlled electric stepping motor was adopted to operate the Ma-s-TS. An electrometer (Keithley 6514) was used to measure the voltage signal. NI USB-6356 (National Instruments Corporation) was used for multi-channel data collection. The software was constructed on LabVIEW platform for real-time data acquisition, analysis and control. A commercial robotic hand (LOBOT uhand, Shenzhen Hiwonder Technology Co., Ltd.) was adopted in the real-time interaction system.

\section{Results and Discussion}

The structure follows a sliding mode as illustrated in Fig. 1. Figure 1a presents the structural scheme of the Ma-s-TS, which consists of an acrylic rectangular cavity and a slider. The multilayer structure from bottom to top is the acrylic 


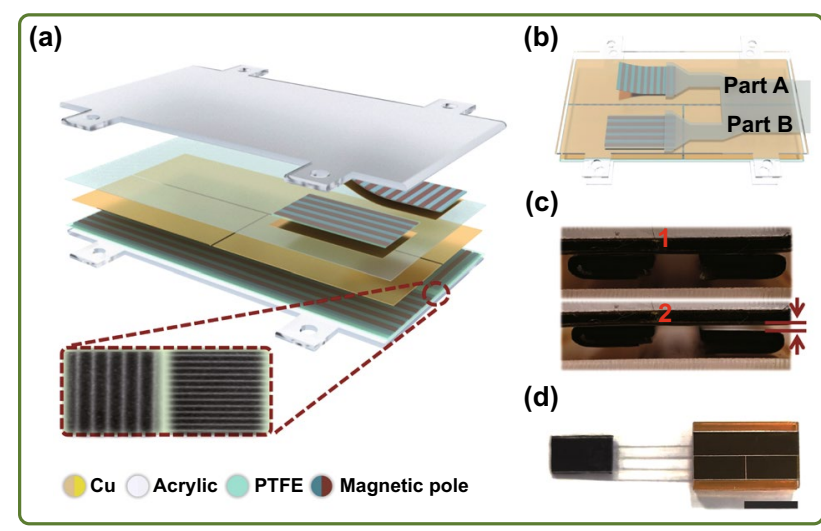

Fig. 1 Structure of the Ma-s-TS. a Schematic diagram and multilayer structure of the Ma-s-TS. The insets: developing photograph of the magnetic array on the substrate and slider. b Overall structure diagram. c Magnetic array assisted contact-separation state (top and bottom). d Photograph of an as-fabricated sensor (scale bar: $1 \mathrm{~cm}$ )

substrate, the magnetic stripe distributed in two vertical directions (part I and part II) in plane, the copper electrode divided into two parts, and the polytetrafluoroethylene (PTFE) surface. The slider also consists of two parts, which from bottom to top is the copper layer and the magnetic stripe distributed in same direction with the stator on each part. The insets show the developing photograph of the magnetic array on the substrate and slider. Left part forms an attractive track for stable sliding, while the right part forms the attraction-repulsion of magnetic pole in sliding. The operating principle is described later in Fig. 2. Figure $1 \mathrm{~b}$ shows the overall structure diagram. Figure 1c shows the contact-separation state of the right part of slider and the stator in sliding process. In this process, the left part of slider is always in attraction state to the stator to form a stable sliding track. Figure 1d shows the as-fabricated Ma-s-TS. The detailed fabrication process is presented in the Experimental Section.

The operating mechanism of the Ma-s-TS is illustrated in Fig. 2. Firstly, electrons transfer from the electrode layer to the FEP film owing to the different triboelectric polarities. And then, for part A, the slider separates from the stator under the repulsive force of the magnetic field when their magnetic properties are identical. Keeping sliding, the slider contacts with the stator under the attraction when their magnetic properties are different. At the same time, for part B, the slider is always attracted to the stator and slides along a straight line due to the magnetic pull. Thus, as shown in Fig. 2a, b, the alternating electric signals from the paired double electrodes in contact-separation state are a series of periodic narrow pulses in part A. For part B, the electrode in free-standing mode generates alternating wide-pulse signals in a sliding cycle. These two kinds of signals are coupled to form a series of positive/negative pulses representing the finger's flexion/extension. To interpret the working principle, the potential distribution of part A electrode (top, Fig. 2c) and part B electrode (bottom, Fig. 2c) under open-circuit condition is simulated by COMSOL in four phases of an ordinary cycle. The actual measured open-circuit voltage of part A and part B and that of the coupled circuit can be referred to Fig. S2. Furthermore, corresponding to Fig. 2b, the load voltage signals out of part A and part B are individually measured to verify the working principle. Figure $2 \mathrm{~d}-\mathrm{f}$ shows the real signals detected from the two independent parts and the coupled circuit.

To quantitatively characterize the performance of the Ma-s-TS, a numerical controlled electric stepping motor is used to drive a hinge component to operate it [15]. To demonstrate the pulse number representing the finger's flexion/ extension degree, the stepping motor is set to rotate through different angles $\left(54^{\circ}, 72^{\circ}\right.$, and $\left.90^{\circ}\right)$ at a rotation speed of $0.375 \mathrm{rps}$. Meanwhile, the sequences of pulses generated by the Ma-s-TS are recorded, as illustrated in Fig. 3a-c, respectively. The generated pulse number is linear to the rotation angle. Therefore, the pulse number represents the finger's flexion/extension degree. To investigate the influence of rotation speed, the stepping motor is set to rotate through $90^{\circ}$ at different rotation speed $(0.375,0.50$, and $0.625 \mathrm{rps})$. From Fig. 3d-f, it can be found that the total pulse number is a constant at the same rotation degree, which means through counting the pulse number the finger's flexion/extension degree can be judged stably/accurately. In addition, through 
(a)
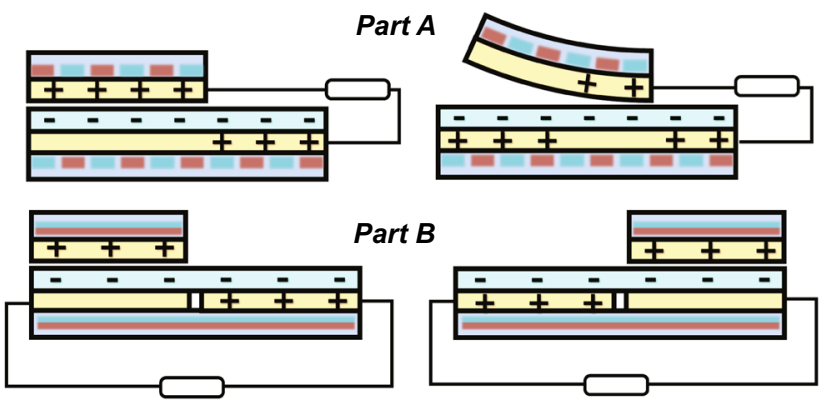

(b)

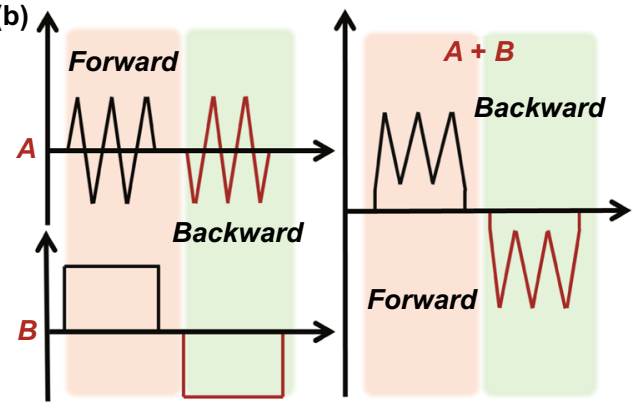

(c)
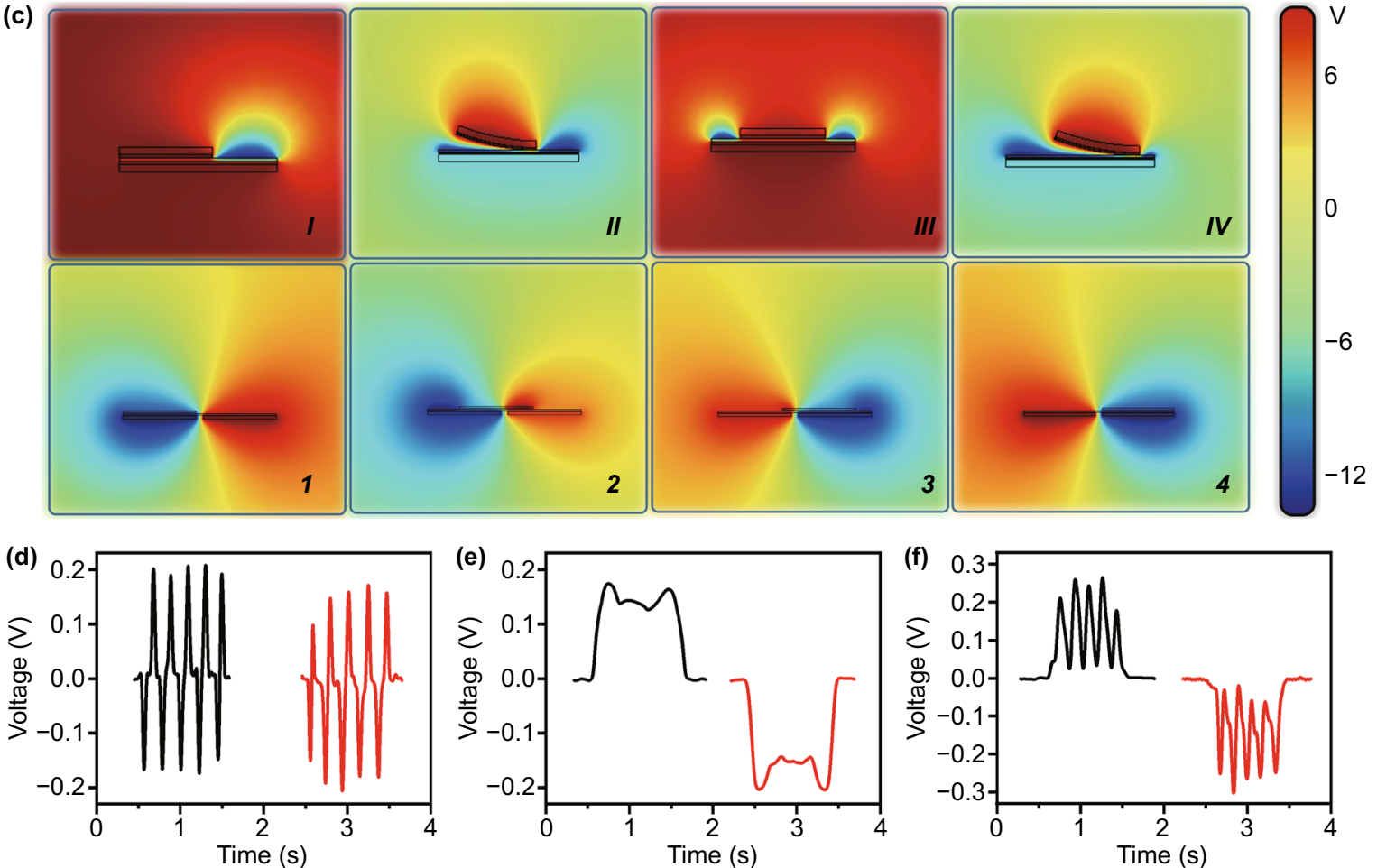

Fig. 2 Operating-principle of the Ma-s-TS. a Schematics of electron transfer process in the sliding. b Schematics of output signal in sliding process. $\mathbf{c}$ COSMOL simulation of potential distribution under various states in sliding process. $\mathbf{d}-\mathbf{f}$ Test output signal corresponding to Fig. $2 \mathrm{~b}$

calculating the number of pulses per unit time, the motion speed can also be determined. The influence of the thickness to sensing accuracy is also tested in Fig. S3, because different thickness of magnetic stripe has different number of pole pairs. Meanwhile, the relationship between driving force and thickness also be explored in Fig. S4.
The output signal is a coupled signal of two parts of electrode. Therefore, the area ratio of the two parts will affect the character of the output signal, hence further affect the backend interaction. The detailed features are studied as shown in Fig. 4. Figure 4a illustrates the area adjustment diagram of the copper electrode of part A and part B, which corresponds 

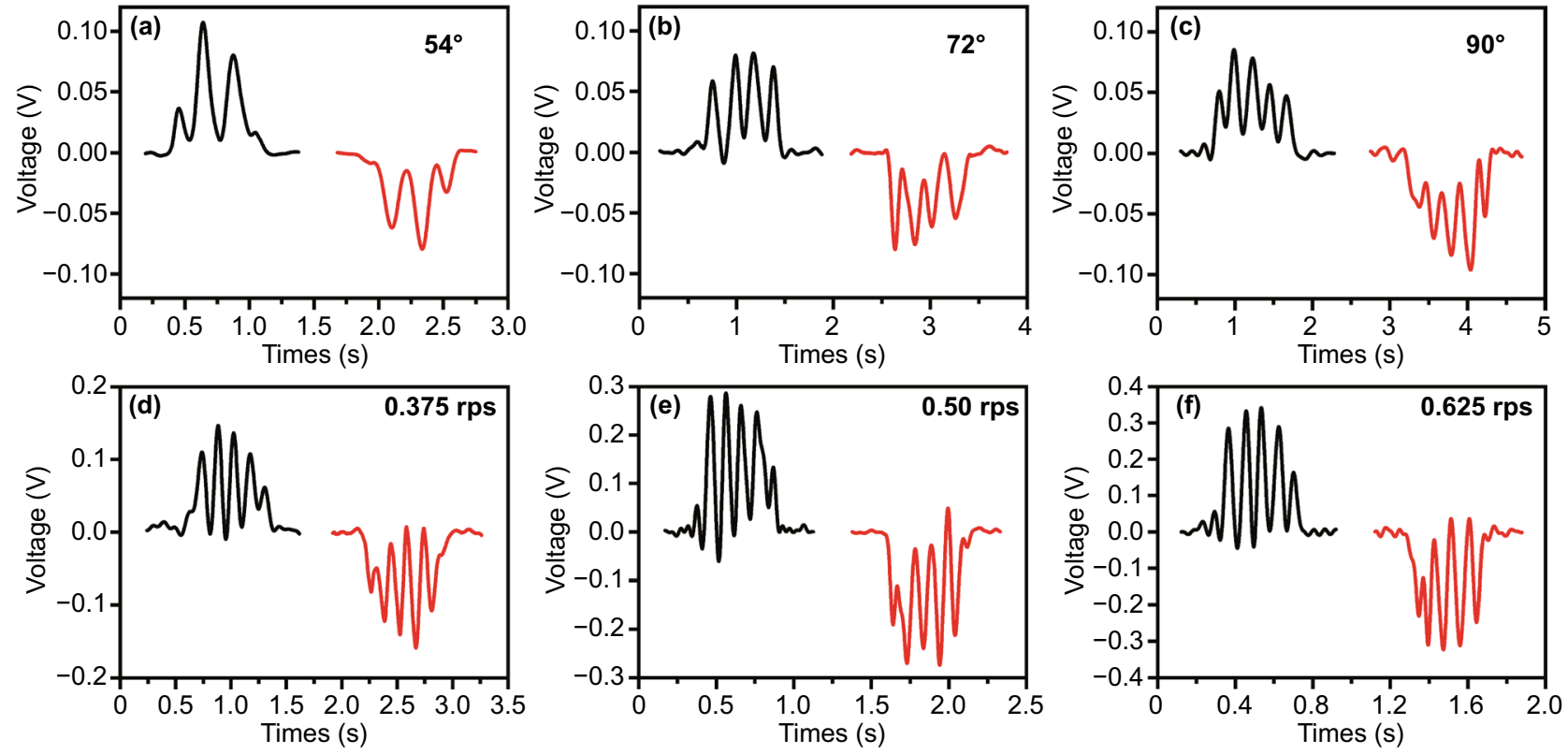

Fig. 3 Characterization of the basic performance of Ma-s-TS. a-c Pulses produced from a Ma-s-TS when rotate through different angles $\left(54^{\circ}\right.$, $72^{\circ}$, and $\left.90^{\circ}\right)$ at rotation speed of $0.375 \mathrm{rps}$. d-f The pulses generated from a Ma-s-TS when rotate through $90^{\circ}$ at different rotation speed $(0.375$, 0.50 , and $0.625 \mathrm{rps})$

to narrow pulses and wide pulses, respectively. According to the test data, as shown in Fig. 4b, when area ratio of part B to part $\mathrm{A}$ increases, the motion speed response range shifts left. To further understand this feature, the test data of an area ratio of 0.8 is illustrated in Fig. $4 \mathrm{c}$. Figure $4 \mathrm{~d}-\mathrm{f}$ is the enlarged signals of Fig. $4 \mathrm{c}$ at a low, medium, and high rotation speed for a better understanding. From rotation speed of $0.25-1.75 \mathrm{rps}$, when the slider moves slowly, the positive/ negative narrow pulses are almost evenly distributed beside baseline and not be biased by the wide pulse from part B, hence hard to be distinguished (as shown in Fig. 4d). When the slider moves fast, the positive/negative narrow pulses are excessively biased by the wide pulse from part B and are hardly distinguished (as shown in Fig. 4f). For different response range requirements, in the state of slow sliding, a bigger area ratio of part $\mathrm{B}$ to part A helps getting a bigger bias (left shifts, Fig. 4b); while in the state of fast sliding, a smaller area ratio of part B to part A helps getting a smaller bias (right shifts, Fig. 4b). An ideal state in Fig. 4e shows that the best response range of the area ratio of 0.8 is around $1.0 \mathrm{rps}$. Therefore, it is necessary to choose the area ratio of the two parts of Ma-s-TS according to the speed response range requirements in different applications.

Based on the features of the Ma-s-TS above, a real-time gesture interaction system is demonstrated as shown in Fig. 5. Figure 5a shows the Ma-s-TS worn on the fingers. In Fig. 5b, the positive/negative pulses represent extending and bending of fingers, respectively. The phases of the humanrobotic hand corresponding to the mark numbers in Fig. 5b are demonstrated in Fig. 5c. The motion of the robotic finger is totally/completely synchronous to the motion of human fingers in real-time. A real-time multi-directional continuous control of the robotic hand is demonstrated in Video S1. In this demonstrations, the real-time control can recover at any breakpoint in finger's flexion/extension process. Besides, Ma-s-TS also showed good stability in multichannel control. 
(a)

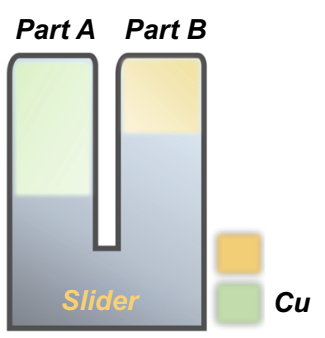

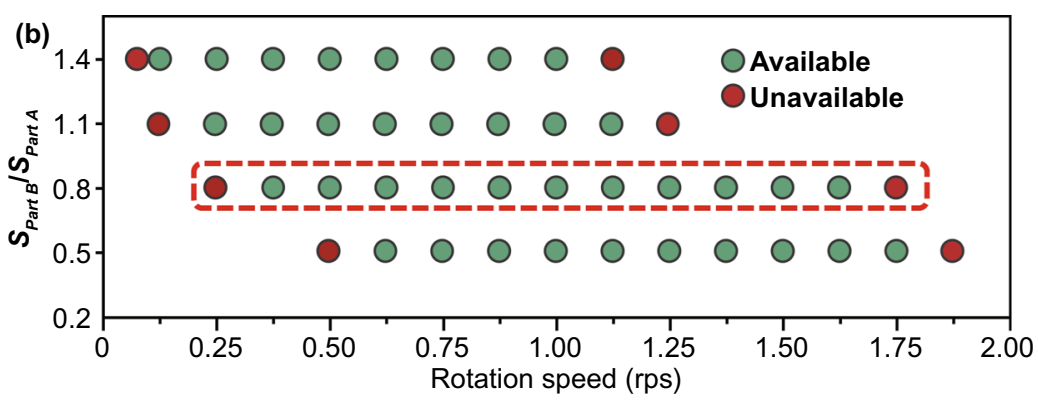

(c)
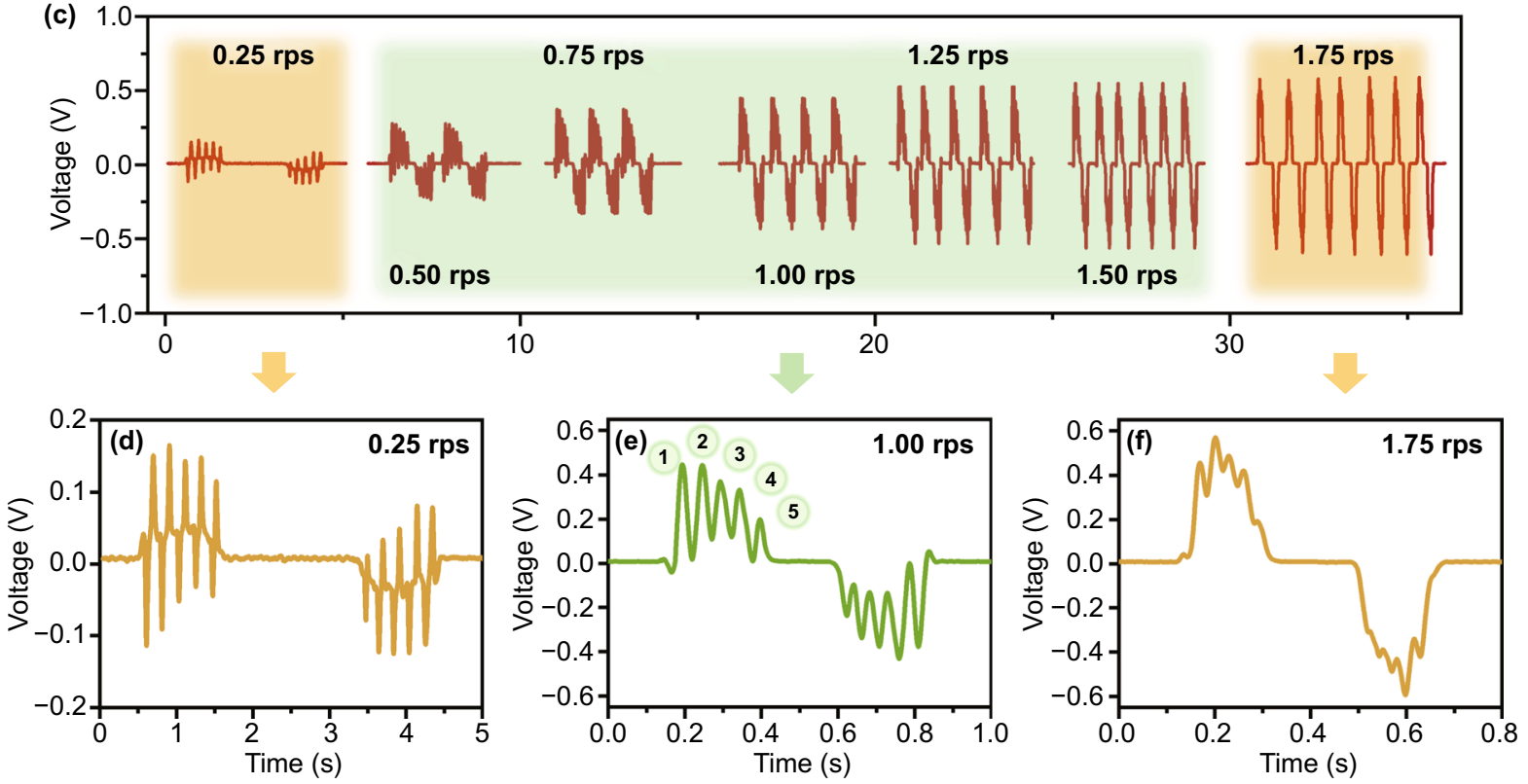

Fig. 4 Matching of two coupled parts of the slider. a Area adjustment diagram of the two parts. b Speed response range under different area ratio of part B to part A. $\mathbf{c}$ Test signals at rotation speed from 0.25 to $1.75 \mathrm{rps}$ under area ratio of 0.8 . d-f Enlarged signal of Fig. $4 \mathrm{c}$ at rotation speed of $0.25,1.00$, and $1.75 \mathrm{rps}$

When some fingers bend, the other fingers are often driven unconsciously. And the unconscious movement will always produce a wrong cross talk signal. As demonstrated in Video S2, Ma-s-TS can suppressed the cross talk between different channels effectively, benefitting from restriction of magnetic force to slider. Here, the cross talk between channels of the Ma-s-TS (Fig. 5e) is compared with previous joint motion triboelectric quantization sensor (Fig. 5d) [15]. The Ma-s-TS is more stable and thus the cross talk caused by finger linkage is suppressed, which is very important for achieving a stable real-time gesture interaction. Futhermore, the durability of Ma-s-TS also has been tested in Fig. S5. After 6,200 operation cycle (at the working frequency of $0.5 \mathrm{~Hz}$ ), the result shows that the normalized output declines to $84.6 \%$ due to intermittent contact. Besides, the output signal of previous work produced continuous narrow pulse signals based on the freestanding model is greatly affected after working for a long time. Hence, we also compared the durability of part A with previous work, as shown in Fig. S5b. 
(a)

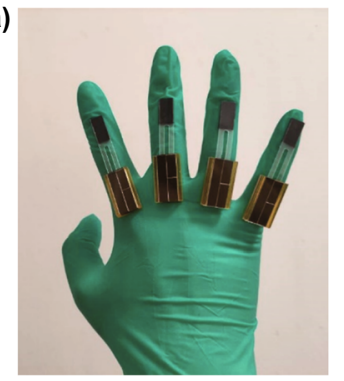

(c)

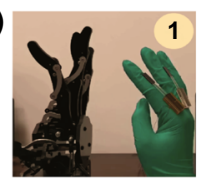

(d)
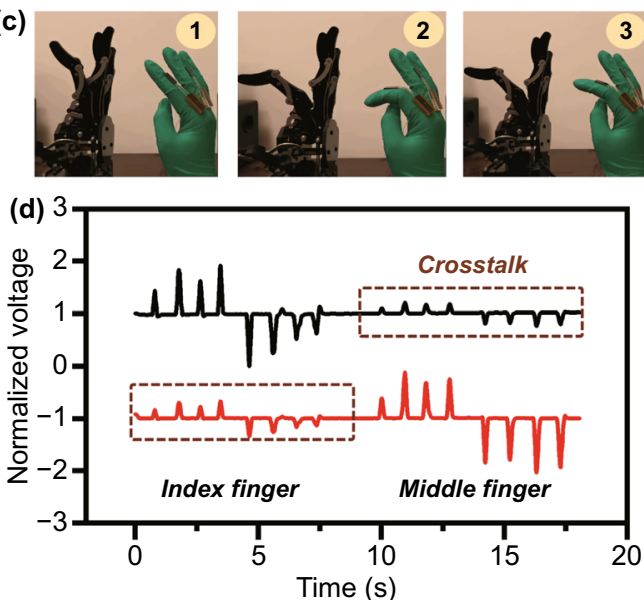

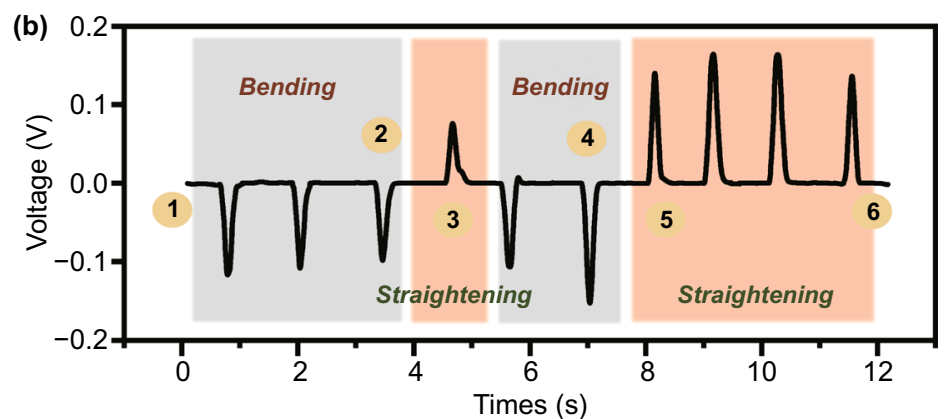

Times (s)
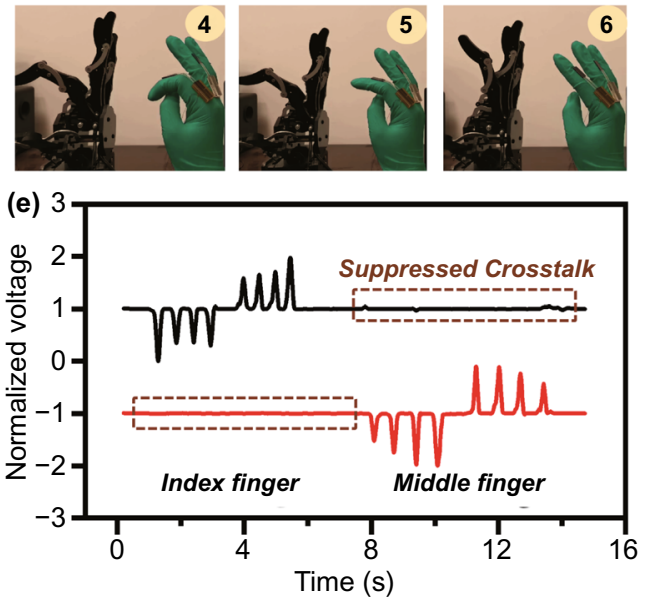

Fig. 5 Real-time gesture interaction demonstration. a Ma-s-TS worn on the fingers. b Positive and negative pulses represent straightening and bending of finger. $\mathbf{c}$ Real-time gesture interaction of robotic hand and human hand based on the signal of Fig. 5b. d-e Comparison with jmTQS [15] on cross talk between channels (d jmTQS, e Ma-s-TS)

\section{Conclusions}

In summary, a magnetic array assisted sliding triboelectric sensor has been proposed for achieving a real-time gesture interaction between a human hand and robotic hand. Through counting the positive/negative pulses that represent extending and bending of fingers, respectively, the degree, speed and direction of the fingers' flexion/extension can be judged.
Besides, the magnetic array assisted sliding structure constrains the sliding direction and translates the sliding motion into contact-separation, which greatly improve the stability, durability and low-speed signal amplitude. Based on these novelties, a real-time gesture interaction system has been established. Furthermore, this Ma-s-TS can be applied in other similar joint's motion detection for a more natural, high-precision and real-time synchronous human-machine interaction. 
Acknowledgements This work was supported by National Natural Science Foundation of China (51902035 and 52073037), Natural Science Foundation of Chongqing (cstc2020jcyj-msxmX0807), the Fundamental Research Funds for the Central Universities (2020CDJ-LHSS-001 and 2019CDXZWL001) and Chongqing graduate tutor team construction project (ydstd1832).

\section{Compliance with Ethical Standards}

Conflict of interest Authors declare that they have no conflict of interest.

Open Access This article is licensed under a Creative Commons Attribution 4.0 International License, which permits use, sharing, adaptation, distribution and reproduction in any medium or format, as long as you give appropriate credit to the original author(s) and the source, provide a link to the Creative Commons licence, and indicate if changes were made. The images or other third party material in this article are included in the article's Creative Commons licence, unless indicated otherwise in a credit line to the material. If material is not included in the article's Creative Commons licence and your intended use is not permitted by statutory regulation or exceeds the permitted use, you will need to obtain permission directly from the copyright holder. To view a copy of this licence, visit http://creativecommons.org/licenses/by/4.0/.

Supplementary information The online version contains supplementary material at (doi:https://doi.org/10.1007/s4082 0-020-00575-2).

\section{References}

1. X.J. Pu, H.Y. Guo, J. Chen, X. Wang, Y. Xi et al., Eye motion triggered self-powered mechnosensational communication system using triboelectric nanogenerator. Sci. Adv. 3(7), e1700694 (2017). https://doi.org/10.1126/sciadv.1700694

2. Y. Kim, A. Chortos, W.T. Xu, Y.X. Liu, J.Y. Oh et al., A bioinspired flexible organic artificial afferent nerve. Science 360(6392), 998-1003 (2018). https://doi.org/10.1126/scien ce.aao0098

3. X. Pu, Q. Tang, W. Chen, Z. Huang, G. Liu et al., Flexible triboelectric 3D touch pad with unit subdivision structure for effective $X Y$ positioning and pressure sensing. Nano Energy 76, 105047 (2020). https://doi.org/10.1016/j.nanoe n. 2020.105047

4. C.R. Qiu, B. Wang, N. Zhang, S.J. Zhang, J.F. Liu et al., Transparent ferroelectric crystals with ultrahigh piezoelectricity. Nature 577(7790), 350-354 (2020). https://doi.org/10.1038/ s41586-019-1891-y

5. S. Takamatsu, T. Lonjaret, E. Ismailova, A. Masuda, T. Itoh et al., Wearable keyboard using conducting polymer electrodes on textiles. Adv. Mater. 28(22), 4485-4488 (2016). https://doi. org/10.1002/adma.201504249

6. A.J. Bandodkar, P. Gutruf, J. Choi, K. Lee, Y. Sekine et al., Battery-free, skin-interfaced microfluidic/electronic systems for simultaneous electrochemical, colorimetric, and volumetric analysis of sweat. Sci. Adv. 5(1), eaav3294 (2019). https://doi.org/10.1126/sciadv.aav3294

7. G.Z. Yang, J. Bellingham, P.E. Dupont, P. Fischer, L. Floridi et al., The grand challenges of science robotics. Sci. Robot. 3(14), eaar7650 (2018). https://doi.org/10.1126/scirobotic s.aar7650

8. M.L. Zhu, Z.D. Sun, Z.X. Zhang, Q.F. Shi, T.Y.Y. He et al., Haptic-feedback smart glove as a creative human-machine interface (HMI) for virtual/augmented reality applications. Sci. Adv. 6(19), eaaz8693 (2020). https://doi.org/10.1126/ sciadv.aaz8693

9. T. D’Orazio, R. Marani, V. Reno, G. Cicirelli, Recent trends in gesture recognition: how depth data has improved classical approaches. Image Vis. Comput. 52, 56-72 (2016). https ://doi.org/10.1016/j.imavis.2016.05.007

10. J.W. Jeong, W.H. Yeo, A. Akhtar, J.J.S. Norton, Y.J. Kwack et al., Materials and optimized designs for human-machine interfaces via epidermal electronics. Adv. Mater. 25(47), 6839-6846 (2013). https://doi.org/10.1002/adma.20130 1921

11. P.G. Jung, G. Lim, S. Kim, K. Kong, A wearable gesture recognition device for detecting muscular activities based on air-pressure sensors. IEEE Transac. Ind. Inform. 11(2), 485-494 (2015). https://doi.org/10.1109/TII.2015.2405413

12. S. Alavi, D. Arsenault, A. Whitehead, Quaternion-based gesture recognition using wireless wearable motion capture sensors. Sensors 16(5), 605 (2016). https://doi.org/10.3390/s1605 0605

13. K. Dong, J.A. Deng, W.B. Ding, A.C. Wang, P.H. Wang et al., Versatile core-sheath yarn for sustainable biomechanical energy harvesting and real-time human-interactive sensing. Adv. Energy Mater. 8(23), 1801114 (2018). https://doi. org/10.1002/aenm.201801114

14. R. Cao, X.J. Pu, X.Y. Du, W. Yang, J.N. Wang et al., Screenprinted washable electronic textiles as self-powered touch/gesture tribo-sensors for intelligent human-machine interaction. ACS Nano 12(6), 5190-5196 (2018). https://doi.org/10.1021/ acsnano.8b02477

15. X. Pu, H. Guo, Q. Tang, J. Chen, L. Feng et al., Rotation sensing and gesture control of a robot joint via triboelectric quantization sensor. Nano Energy 54, 453-460 (2018). https ://doi.org/10.1016/j.nanoen.2018.10.044

16. L. Xie, X. Chen, Z. Wen, Y. Yang, J. Shi et al., Spiral steel wire based fiber-shaped stretchable and tailorable triboelectric nanogenerator for wearable power source and active gesture sensor. Nano-Micro Lett. 11(1), 39 (2019). https://doi. org/10.1007/s40820-019-0271-3

17. F. Wen, Z.D. Sun, T.Y.Y. He, Q.F. Shi, M.L. Zhu et al., Machine learning glove using self-powered conductive superhydrophobic triboelectric textile for gesture recognition in VR/AR applications. Adv. Sci. 7(14), 2000261 (2020). https ://doi.org/10.1002/advs.202000261

18. Q. Tang, X.J. Pu, Q.X. Zeng, H.M. Yang, J. Li et al., A strategy to promote efficiency and durability for sliding energy 
harvesting by designing alternating magnetic stripe arrays in triboelectric nanogenerator. Nano Energy 66, 104087 (2019). https://doi.org/10.1016/j.nanoen.2019.104087

19. D. Liu, X. Yin, H.Y. Guo, L.L. Zhou, X.Y. Li et al., A constant current triboelectric nanogenerator arising from electrostatic breakdown. Sci. Adv. 5(4), eaav6437 (2019). https://doi. org/10.1126/sciadv.aav6437

20. Z.L. Wang, A.C. Wang, On the origin of contact-electrification. Mater. Today 30, 34-51 (2019). https://doi.org/10.1016/j. mattod.2019.05.016

21. X.X. Chen, Z.Y. Ren, H. Guo, X.L. Cheng, H.X. Zhang, Selfpowered flexible and transparent smart patch for temperature sensing. Appl. Phys. Lett. 116(4), 043902 (2020). https://doi. org/10.1063/1.5134526

22. Q. Tang, M.H. Yeh, G. Liu, S. Li, J. Chen et al., Whirligiginspired triboelectric nanogenerator with ultrahigh specific output as reliable portable instant power supply for personal health monitoring devices. Nano Energy 47, 74-80 (2018). https://doi.org/10.1016/j.nanoen.2018.02.039

23. Z. Liu, H. Li, B.J. Shi, Y.B. Fan, Z.L. Wang, Z. Li, Wearable and implantable triboelectric nanogenerators. Adv. Funct.
Mater. 29(20), 1808820 (2019). https://doi.org/10.1002/ adfm.201808820

24. H.Y. Guo, X.J. Pu, J. Chen, Y. Meng, M.H. Yeh et al., A highly sensitive, self-powered triboelectric auditory sensor for social robotics and hearing aids. Sci. Robot. 3(20), eaat2516 (2018). https://doi.org/10.1126/scirobotics.aat2516

25. G.F. Cai, J.H. Ciou, Y.Z. Liu, Y. Jiang, P.S. Lee, Leaf-inspired multiresponsive MXene-based actuator for programmable smart devices. Sci. Adv. 5(7), eaaw7956 (2019). https://doi. org/10.1126/sciadv.aaw7956

26. G. Liu, L. Xiao, C. Chen, W. Liu, X. Pu et al., Power cables for triboelectric nanogenerator networks for large-scale blue energy harvesting. Nano Energy 75, 104975 (2020). https:// doi.org/10.1016/j.nanoen.2020.104975

27. Z.M. Wang, J. An, J.H. Nie, J.J. Luo, J.J. Shao et al., A Self-powered angle sensor at nanoradian-resolution for robotic arms and personalized medicare. Adv. Mater. 32(32), 2001466 (2020). https://doi.org/10.1002/ adma.202001466 